\title{
SAND2000-0849C
}

\section{Recent Advances in High-Pressure Equation-of-State Capabilities*}

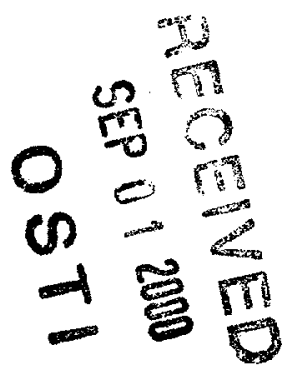

James R. Asay, Clint A. Hall, Marcus Knudson

Sandia National Laboratories, Albuquerque, NM 87185

For many scientific and programmatic applications, it is necessary to determine the shock compression response of materials to several tens of Mbar. In addition, a complete EOS is often needed in these applications, which requires that shock data be supplemented with other information, such as temperature measurements or by EOS data off the principal Hugoniot. Recent developments in the use of fast pulsed power techniques for EOS studies have been useful in achieving these goals. In particular, the $Z$ accelerator at Sandia National Laboratories, which develops over 20 million amperes of current in 100-200 ns, can be used to produce multi-Mbar shock pressures and to obtain continuous compression data to pressures exceeding $1 \mathrm{Mbar}$. With this technique, isentropic compression data have been obtained on several materials to pressures of several hundred kbar. The technique has also been used to launch ultra-high velocity flyer plates to a maximum velocity of $14 \mathrm{~km} / \mathrm{s}$, which can be used to produce impact pressures of several Mbar in low impedance materials and over $10 \mathrm{Mbar}$ in high impedance materials. The paper will review developments in both of these areas.

\section{Introduction}

The high-pressure equation-of-state (EOS) of materials is normally determined experimentally using shock loading techniques [1]. In these methods, flat cylindrical specimens are subjected to planar shock loading for time durations of a few hundred nanoseconds. Typical time durations are several microseconds with resultant steady shock properties measured, such as the shock velocity and particle velocity behind the shock. The shock Hugoniot jump conditions are used with these data to determine the pressure-volume-energy response of the material along a path referred to as the Hugoniot, which is the locus of end states produced by steady shock compression [1]. Shock wave techniques are useful for generating high-pressure EOS properties, but the Hugoniot curve is not sufficient to determine a complete pressure-volumetemperature surface, which normally requires material response along thermodynamic paths lying off the Hugoniot. [1]. A specific off-Hugoniot path is the compression isentrope at room temperature, which is the subject of this paper.

In the following sections, a brief review of experimental approaches for measuring the highpressure isentropic response of materials is presented. A magnetic loading capability recently developed for producing isentropic compression to Mbar pressures is then discussed, with examples of recent data obtained on copper and other materials. An extension of the isentropic loading technique involves the application to launching relatively thick flyer plates under shockless loading conditions to velocities far exceeding conventional gas gun

"Sandia is a multi-program laboratory operated by Sandia Corporation, a Lockheed Martin Company, for the United States Department of Energy under Contract DE-AC04-94AL85000. 


\section{DISCLAIMER}

This report was prepared as an account of work sponsored by an agency of the United States Government. Neither the United States Government nor any agency thereof, nor any of their employees, make any warranty, express or implied, or assumes any legal liability or responsibility for the accuracy, completeness, or usefulness of any information, apparatus, product, or process disclosed, or represents that its use would not infringe privately owned rights. Reference herein to any specific commercial product, process, or service by trade name, trademark, manufacturer, or otherwise does not necessarily constitute or imply its endorsement, recommendation, or favoring by the United States Government or any agency thereof. The views and opinions of authors expressed herein do not necessarily state or reflect those of the United States Government or any agency thereof. 


\section{DISCLAIMER}

Portions of this document may be illegible in electronic image products. Images are produced from the best available original document. 
capabilities. This technique will also be discussed, with examples given for copper, aluminum and titanium. The ability to launch ambient flyer plates to velocities in the $10-20 \mathrm{~km} / \mathrm{s}$ range significantly expands the peak shock pressures accessible in the laboratory for EOS applications.

\section{Background}

The relationship between the isotherm, the principal Hugoniot, and the room temperature isentrope is shown in Fig. 1 for aluminum. The isentrope, which lies between the isotherm and the Hugoniot curve, represents the response obtained for continuous, adiabatic and reversible compression, whereas the Hugoniot is the locus of end states achieved by single shock compression. The isentrope and isotherm are nearly identical to pressures approaching $1 \mathrm{Mbar}$, since the temperature increase produced during isentrope compression is small [1]. Furthermore, the

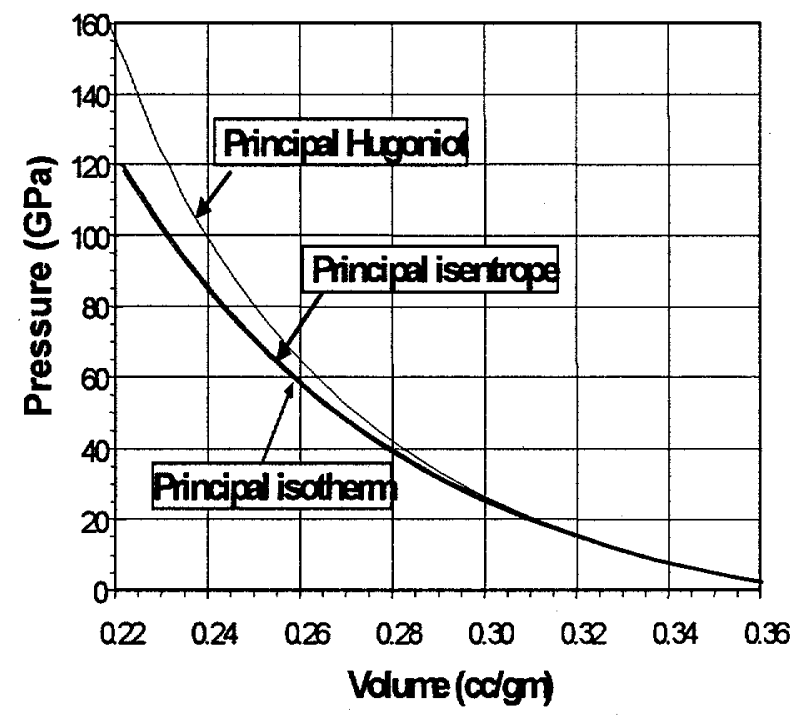

Figure 1. Relationship between the isotherm, isentrope and Hugoniot

isentrope and the Hugoniot are second-order tangent at the initial volume state [2], so all three curves are nearly coincident to pressures of about $300 \mathrm{kbar}$. It is extremely difficult to produce states of isentropic compression using static methods. However, this is possible with dynamic loading, although the compression is not reversible because of elastic-plastic and dissipative viscoplastic processes occurring during loading. For this reason, ramp or continuous loading is often referred to as quasi-isentropic. However, for the purpose of this paper, ramp loading is referred to as the Isentropic Compression Experiment (ICE).

High-velocity gas guns are often used to produce precision loading of solids and liquids to high pressure. In these techniques, a flat plate is launched to high velocity and impacts a flat disk, as shown in Fig. 2a. At high pressures, a planar shock is produced directly upon impact, with the pressure amplitude determined by the initial impact velocity. Each experiment produces a unique state of pressure, volume and internal energy, which can be determined from the conservation relations.
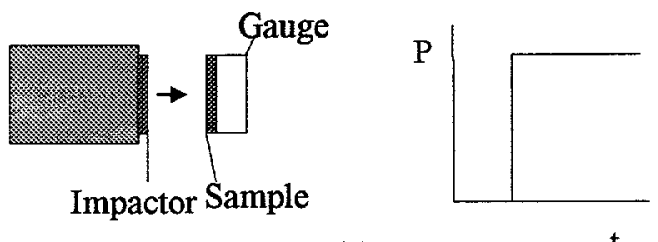

(a)
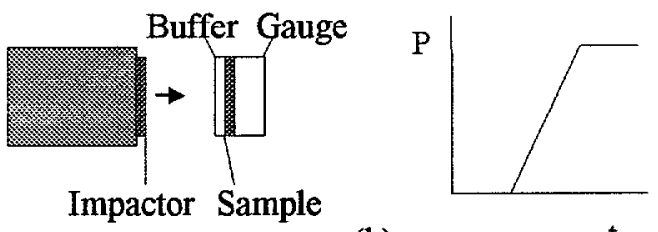

(b)

t

Figure 2. Plate impact techniques for producing (a) shock loading of specimens, and (b) ramp or isentropic loading.

Measurement of two variables in the shock wave, typically the shock velocity and particle velocity, allow determination of the five thermodynamic variables describing the Hugoniot state:

$$
\sigma=\rho_{0} U_{s} u
$$

$$
\begin{aligned}
& \frac{\rho_{0}}{\rho}=1-\frac{u}{U_{s}} \\
& E-E_{0}=\frac{1}{2} \sigma\left(V_{0}-V\right),
\end{aligned}
$$


where $\sigma$ is stress in the shocked state, $u$ is the particle velocity behind the shock front, $U_{s}$ is the shock velocity, $\rho$ is density (reciprocal specific volume, V) and $E$ is specific energy in the shocked state.

Projectile impact techniques have been previously used to study a large number of materials to shock pressures of several Mbar [1]. However, they have not been generally used for producing continuous pressure loading. Barker and Hollenbach [3] used a ramp wave generator, fused silica, to produce isentropic compression of samples attached to the rear surface of the fused silica up to impact stresses of about $30 \mathrm{kbar}$. The risetime of the pressure loading on the specimen was determined by the thickness of the fused silica buffer used. The concept is shown in figure $2 \mathrm{~b}$. In addition to EOS measurements, ramp loading can be used for a variety of other applications, such as studying the dependence of mechanical properties on loading rate [4].

Fused silica ramp generators are limited to loading stresses of about $30 \mathrm{kbar}$. Other gun techniques have been used to generate ramp waves to higher pressure. Asay and Chhabildas [5] used a commercially available ceramic to produce shockless loading in aluminum to shock stresses of about 200 kbar. Ramp wave profiles were obtained on several different sample thicknesses of aluminum, resulting in the generation of a compression isentrope to about $200 \mathrm{kbar}$. Other gas gun techniques include the use of graded density impactors [6] to produce initial small amplitude shocks in a specimen, followed by nearly isentropic compression to Mbar pressures.

Once ramp, or ICE waves, are produced in a specimen, they must be analyzed to determine the stress-density loading curve. An extensive set of analysis methods, generally falling under the category of Lagrangian wave analyses, have been developed for this purpose [7]. If the material response is rate dependent, the analysis process is rather complex, requiring both stress and particle velocity profiles at several propagation distances. However, if the response is rate independent, the resulting wave motion is self-similar, with the motion commonly referred to as simple waves. In this special case, the resulting particle velocity profiles can be analyzed as follows [7]:

$\mathrm{d} \sigma=\rho_{0} \mathrm{c}_{\mathrm{l}}(\mathrm{u}) \mathrm{du}$,

$\mathrm{dV}=\mathrm{V}_{0} \mathrm{du} / \mathrm{c}_{\mathrm{l}}(\mathrm{u})$

$\mathrm{dE}=1 / 2 \sigma(\mathrm{V}) \mathrm{dV}$,

where $\sigma$ is the longitudinal stress component, $\rho_{0}$ is initial density $\left(1 / \mathrm{V}_{0}\right), c_{1}(u)$ is the Lagrangian wave velocity for a given particle velocity, $u$, in the wave profile, and $\mathrm{E}$ is specific energy along the isentropic loading path.

Implementation of Equations 4-6 requires accurate measurements of either a particle velocity-time profile or a stress-time profile, along with measurements of the velocity of the wave at the corresponding particle velocity or stress. Once the profiles are established to be self-similar, it is then possible to integrate the equations given above to determine a continuous stress-density-loading curve. For the data presented later in this paper, the measured wave profiles were judged to be selfsimilar to within experimental uncertainties.

\section{Z Accelerator}

Pulsed energy sources provide an alternative to producing shockless planar loading of specimens. This has been recently demonstrated on the $Z$ Accelerator [8] at Sandia National Laboratories, and has considerable promise for producing smooth compression to Mbar pressures. The $Z$ Accelerator, which is illustrated schematically in Figure 3 , is a low inductance pulsed power generator capable of capacitively storing about $11.6 \mathrm{MJ}$ of electrical energy and produces peak currents of $22 \mathrm{MA}$. The system has been used for a variety of applications, including acceleration of electrons and ions for inertial confinement fusion applications and generation of intense $x$-ray environments through $Z$-pinch driven plasma implosions [8]. In the present application, it is used strictly in the current generation mode, which can be used to magnetically load specimens to high pressure. 
Our goals for isentropic compression experiments on the Accelerator are to obtain accurate continuous

\section{Target Chamber}

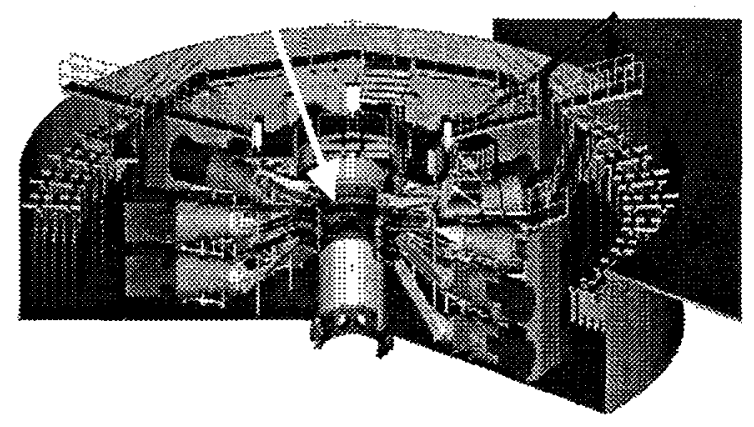

Figure 3. Schematic of the $Z$ Accelerator.

compression data approximating isentropic response. Several specific requirements must be met to achieve this goal:

- One-dimensional, planar pressure loading uniform to within $0.5 \%$ over the sample diameter, which is necessary for planar wave analysis,

- smoothly increasing pressure loading applied to the sample surface over a time scale of $100-200 \mathrm{~ns}$, which is necessary to prevent shock formation,

- propagation of compression waves into undisturbed material at a known initial state for accurate application of the wave analyses given earlier,

- study of a minimum of two samples experiencing "identical" loading for evaluation of self-similar motion and use of the Lagrangian analysis method,

- measurement of time-resolved particle velocity histories at the rear surface of specimens with $1 \%$ accuracy in wave speeds.

The technique for magnetically loading specimens is illustrated in Fig.4a. A short circuit is created between the anode and cathode in the $Z$ Accelerator that results in a current flow on the inner surface of each conductor with a magnetic field in the gap separating the two. For the planar conductors shown in the figure, a uniform magnetic field is produced, resulting in essentially constant pressure loading over the front surface of a specimen placed in the anode. Extensive 2-D and 3-D MHD simulations have shown that the magnetic field in the gap is very uniform for the designs used for ICE, resulting in extremely uniform and planar pressure on the sample. A typical current profile produced in the Accelerator is an S-shaped curve peaking at about $20 \mathrm{MA}$ with a risetime on the order of 100-200 ns, as shown in Fig. $4 \mathrm{~b}$. The interaction between the current flow and the magnetic field induces a magnetic pressure on the inner surface of the conducting anode through the relation:

$$
\begin{aligned}
P(t) & =1 / 2 \mu_{0} B^{2}(t), \\
& =1 / 2 \mu_{0} J^{2}(t),
\end{aligned}
$$

where $\mu_{0}$ is the permeability of free space, $\mathbf{J}$ is current density, and B is applied magnetic field.

Considerable progress has been made in achieving the goals for accurate isentropic compression experiments, as discussed by Asay [9], Hall et al. $[10,11]$ and Reisman et al. [12]. A major thrust of this research has been directed to the development of a versatile magnetic loading technique that can be used to produce planar loading conditions in a variety of materials to pressures of several hundred kbar. This has been accomplished by fabricating the anode-cathode geometry from removable panels that contain the specimens and are assembled into the anode of the Accelerator [11]. A photograph of an assembled anode-cathode arrangement is shown in Figure 5.

These investigations have also shown that it is possible to achieve planar loading of specimens about $6 \mathrm{~mm}$ in diameter and a few hundred microns thick over time scales of about $200 \mathrm{~ns}$ and to peak stresses of several hundred kbar. These dimensions allow sufficiently long transit times to achieve the $1 \%$ accuracy in wave speeds at various points on the wave profiles. The other requirements discussed above have been similarly achieved through a 


\section{Time-resolved velocity interferometry}

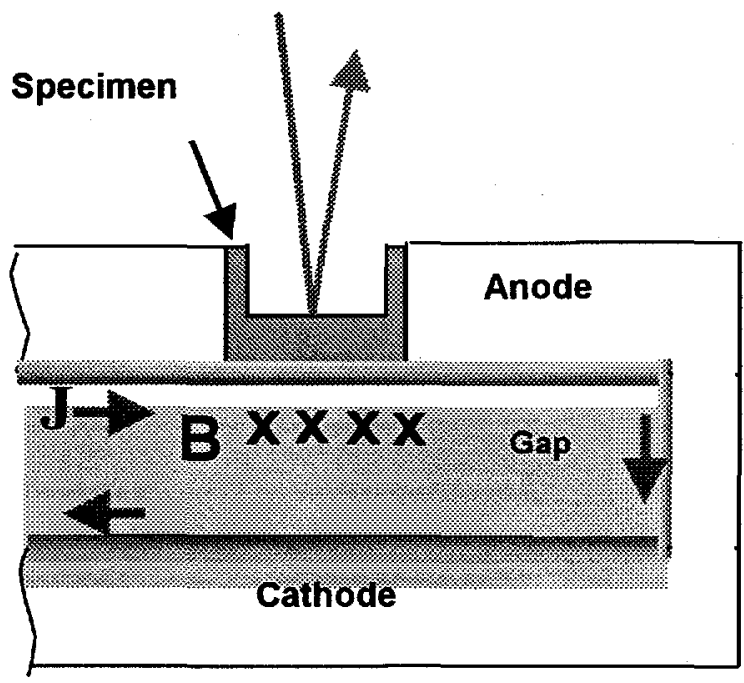

(a)

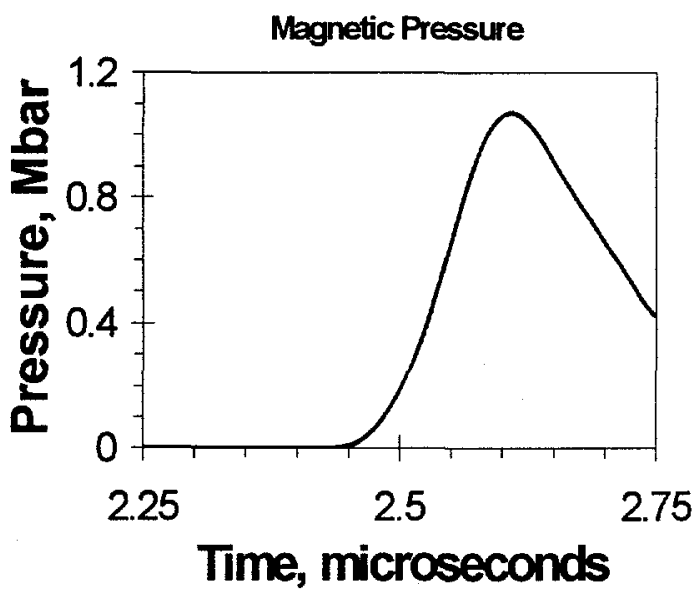

(b)

Figure 4. Geometry for producing planar magnetic loading on samples. (a) Experimental technique, (b) typical pressure input history. combination of experimental and numerical calculations.

In isentropic compression experiments performed to date on the $Z$ Accelerator, samples were fabricated with diamond-machining processes into disks that were about $6 \mathrm{~mm}$ in diameter and several hundred microns thick. The typical tolerance on surface flatness of the samples was about $20 \mathrm{~nm}$. For the case of aluminum or copper samples, the samples were exposed directly to the magnetic field. Other sample materials were mounted on either aluminum or copper conductors, which resulted in mechanical loading of the sample. Extensive MHD calculations that were performed for this experimental configuration indicate that the magnetic field diffuse into the conductor at a rate slower than hydrodynamic wave motion [9], so that the ICE wave produced in this process always evaluates undisturbed material. MHD simulations of the experimental design [12] also demonstrated that pressure variations across the input surface of the sample were typically less than $1 \%$. These predictions have been confirmed to within experimental resolution [11].

Time-resolved velocity interferometry, VISAR [9$11]$, was used to record the rear surface velocity history of two or more samples during a specific experiment. The rear surface velocity profiles from one of the experiments performed on the Accelerator is shown in Figure 6. The figure shows the input current history and the resulting velocity profiles at two propagation distances of about 0.5 $\mathrm{mm}$ and $0.8 \mathrm{~mm}$. A portion of the wave has almost developed into a shock for the $0.8 \mathrm{~mm}$ thick sample, but continues to have a finite, measurable risetime so that it can be used with the equations of motion previously discussed for isentropic compression analysis.

Before the Lagrangian wave speed analysis can be applied to the measured profiles, it is necessary to estimate the effects of the wave perturbation that occurs near the free surface. Since these interactions are quite complex, they require an iterative process to converge on the estimated in- 


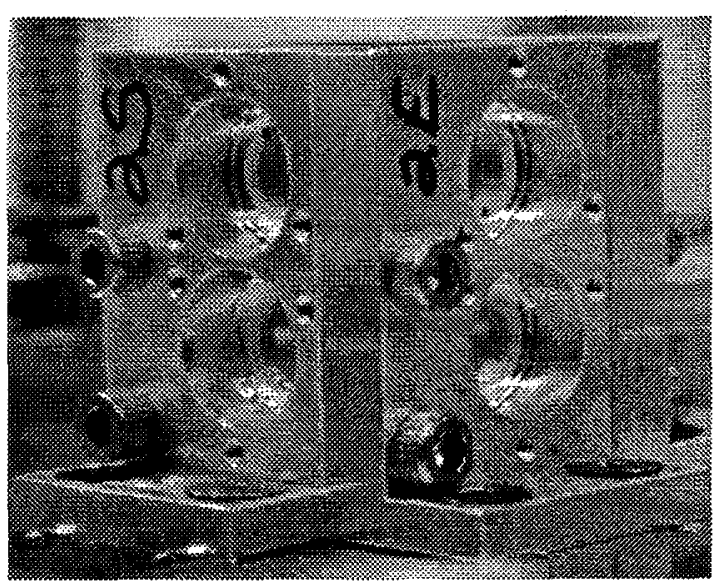

(a)

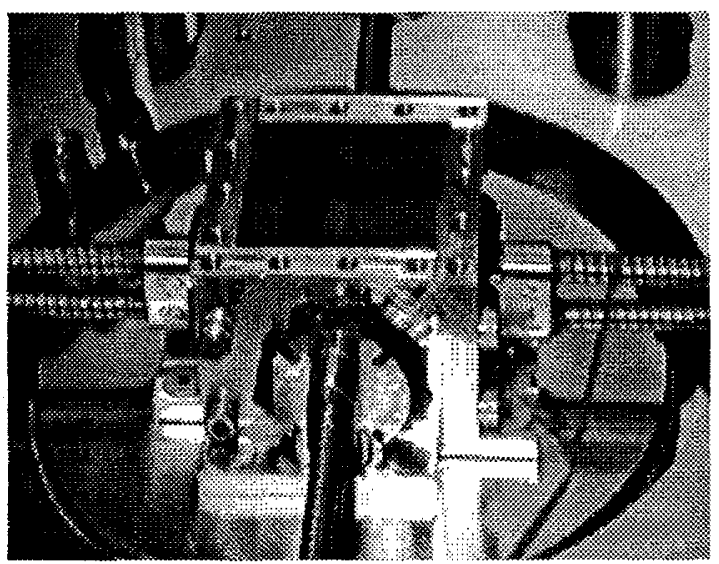

(b)

Figure 5. Photograph of the anode-cathode geometry for producing planar magnetic loading of several samples simultaneously, (a). Two samples are located in the recesses on each of the four panels making up the anode. Fiber optic VISAR connections are shown that measure the response of each sample (b) [11].

situ profiles. This was accomplished by calculating the free surface and in situ particle velocity histories at each free surface, using an assumed EOS for copper, applying a $u_{\mathrm{s}} / 2$ approximation (which assumes that the in situ velocity is exactly one half

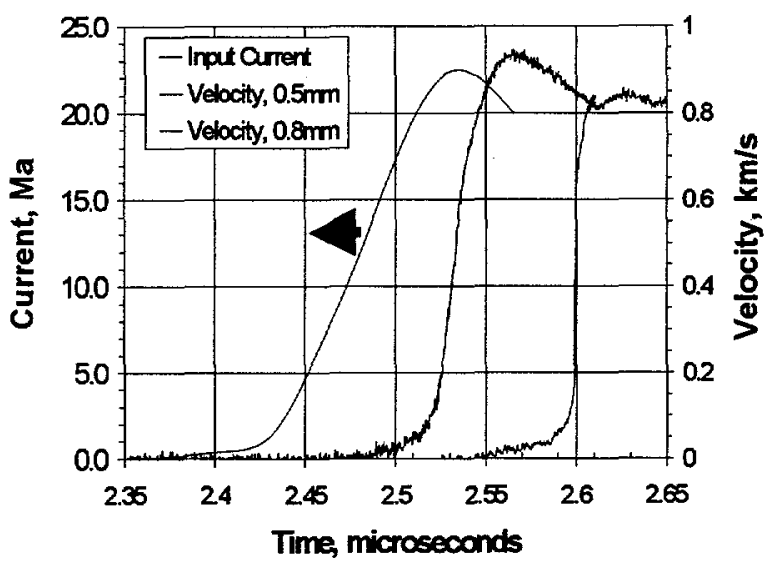

Figure 6. Input current history and particle velocity records obtained on copper specimens nominally 0.5 and $0.8 \mathrm{~mm}$ thick [11].

the measured free-surface velocity), and comparing the result to wave profile calculations at the same sample thicknesses within infinitely thick samples $[11,12]$. Reisman has shown that this process produces a linearly increasing correction to about $2 \%$ in measured transit times for copper isentropically compressed to $200 \mathrm{kbar}$ [12].

Once the measured free surface velocity profiles are corrected for free surface perturbations, equations 4 and 5 are applied to the measured wave and particle speeds to estimate the stress-volume loading curve. The results of several experiments on copper are shown in Figure 7 [12]. Also shown are Hugoniot data from several sources, as reported by Reisman [12]. As can be seen, one isentropic loading experiment can be used to determine a large portion of the isentropic response, whereas several Hugoniot experiments are necessary to define a similar pressure-density relation. It should be noted that the Hugoniot and isentrope are coincident to within about $1 \%$ over this stress regime [10], so that comparisons between shock loading and isentropic response are meaningful at these pressure levels. The error bars shown for the ICE data were based on a careful analysis of the various random errors occurring in the experiment that result in uncertainties of about $2.5 \%$ [12]. 
Another example serves to illustrate the potential of the technique. Reisman et al. [12] present several applications of the technique, including beryllium,

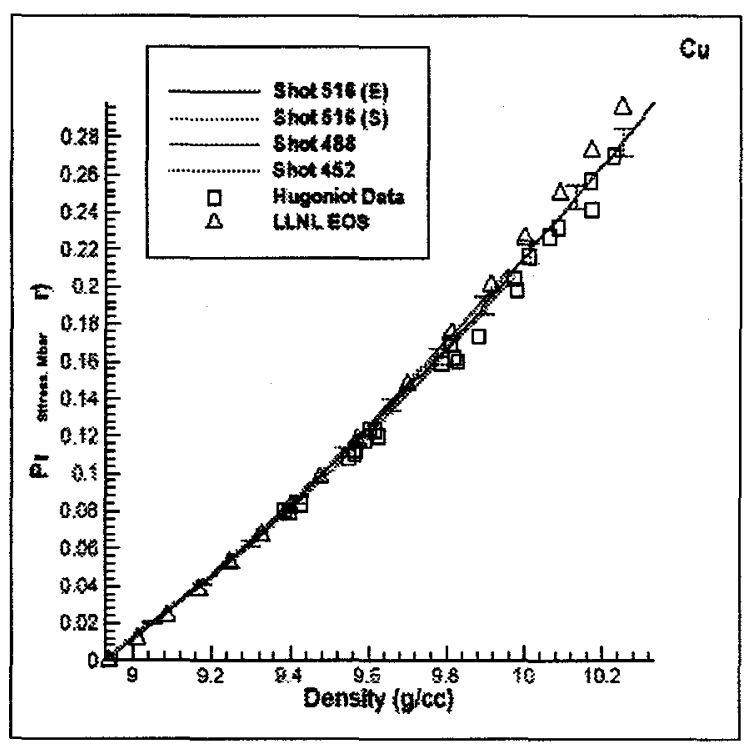

Figure 7. Stress-density loading curve for copper with Hugoniot data over the same stress region [12].

tantalum and molybdenum. The results for beryllium are given in Figure 8. These experiments were performed on sample thicknesses ranging from 300 to $1400 \mu \mathrm{m}$. The samples had average grain sizes of about 50-70 microns, which likely influenced the measured wave profiles because of the large degree of crystalline anisotropy in beryllium. This effect was offset to a large extent by choosing sample pairs of 900 and $1400 \mu \mathrm{m}$ for analyzing the ICE-wave data.

The isentropic compression data shown in Figure 8 are compared to both Hugoniot and isothermal data. To within the reported error bars, there is good agreement between the different techniques, which is expected over this stress regime. Further details can be found in the papers by Hall [10] and Reisman [12].

\section{Application to Phase Transition Studies}

In addition to providing a method for determining the $\mathrm{P}-\mathrm{V}$ response of materials along the isentrope,

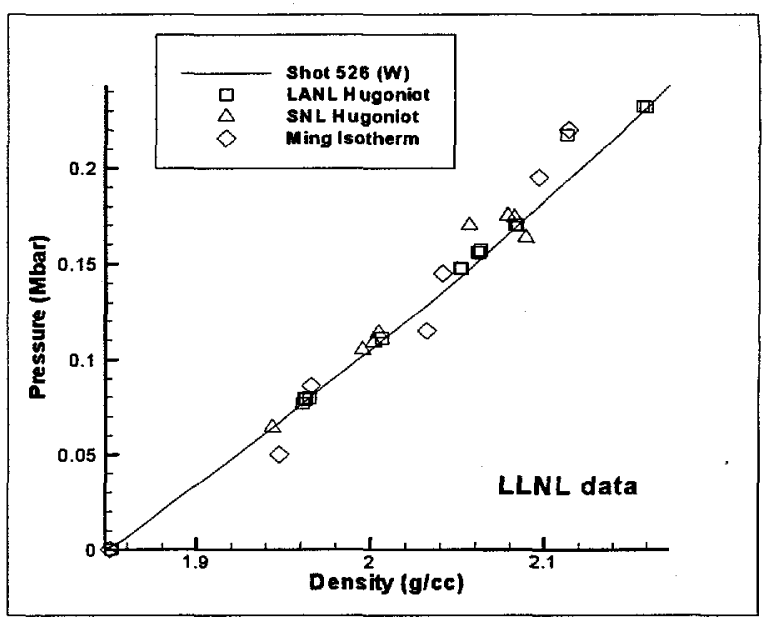

Figure 8. Stress-density loading curve for beryllium obtained with the ICE technique [12].

the ICE method allows detection of polymorphic phase transitions and also determination of kinetics associated with the transition more easily than possible with traditional shock wave techniques. To demonstrate the effect, the method was applied to continuous loading of Armco iron to pressures of about $300 \mathrm{kbar}$. Iron has a well-established solidsolid phase transition from the bcc ( $\alpha)$ to the hcp ( $\varepsilon$ ) phase that occurs at approximately $130 \mathrm{kbar}$. The transition has been investigated by Barker using wave profile techniques on smooth bore launchers [13]. One difficulty in studying phase transitions with shock techniques is that the loading curve followed by shock compression, or Rayleigh line, can easily cause over-driving of a small-volume phase change. This results in no detectable effect on the shock wave. Furthermore, several experiments at varying sample thickness are required to study transformation kinetics of these transitions. Using shock techniques, Barker demonstrated that the transition in iron is not instantaneous under shock loading, but instead has a transition time of approximately $40 \mathrm{~ns}$ [13].

The ICE technique discussed earlier was used with some modification [14] to study the phase transition behavior in iron. ICE-wave profiles for two samples, 0.5 and $0.8 \mathrm{~mm}$ in thickness, were studied using an initial input loading time of about $100 \mathrm{~ns}$. 


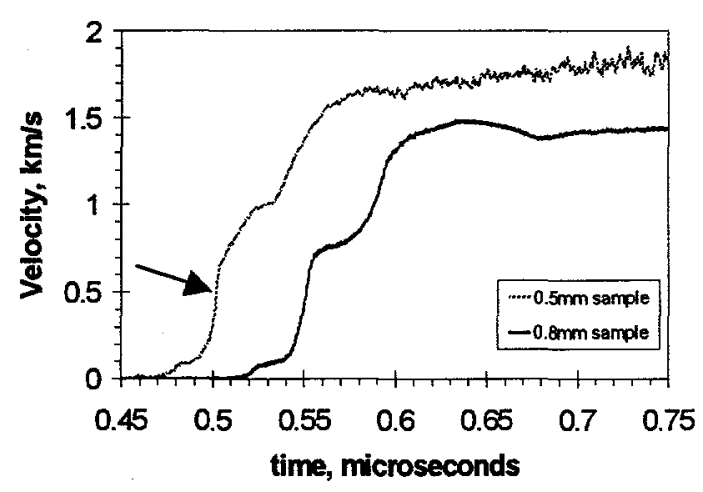

Figure 9. Ramp-wave profiles obtained on iron specimens, 0.5 and $0.8 \mathrm{~mm}$ thick.

The two free surface velocity profiles recorded during this experiment are shown in Figure 9. The elastic precursor is clearly observed in the lower pressure region of the two profiles. Calculation of the elastic wave speed from these profile results in a velocity of $6.24 \mathrm{~km} / \mathrm{s}$, which is in good agreement with reported values of $6.36 \mathrm{~km} / \mathrm{s}$, obtained by Barker [13]. Of greater interest, however, is the region where the phase transition is occurring. Since the loading response actually follows the P-V response of the material, the actual evolution of the phase transition can be directly observed. The velocity profile for the $0.5 \mathrm{~mm}$ thick iron sample in Fig. 9 has an arrow indicating the onset of the phase transition before arrival of the wave traveling in the hcp phase material. The $0.8 \mathrm{~mm}$ thick sample has a fully evolved two-wave structure that more closely resembles results obtained from typical plate impact experiments.

From these profiles it is possible to estimate the kinetics of the phase transition in iron [14]. The theoretical approach employs construction of thermodynamically complete equations of state for both phases, with a transition rate defined by the difference in Gibbs potential in metastable regions, divided by a single relaxation time. The results of this study indicate that an approximate value for the transition time is about $40 \mathrm{~ns}$ [14], which is in good agreement with values obtained by Barker [13] in a larger number of shock experiments. It is important to note from the initial requirements stated for ICE that the results obtained on iron do not represent isentropic response.

\section{Extension to High Velocity Flyer Plate Launch}

An extension of the isentropic loading technique involves the capability to launch flyer plates of a few hundred microns in thickness to velocities exceeding that possible with conventional light gas guns [15]. The magnetic method for launching a flyer plate is essentially identical to the technique represented in Figure 4, except that the flyer thickness is chosen so that wave reverberation times are small compared to the application time for the current. The concept is illustrated in Figure 10.

For the experimental geometry illustrated in Figure 10 and a time-resolved pressure pulse of about 150 ns in duration, a net momentum increase is imparted to the flyer plate, which results in a given velocity for a specific plate mass. The final velocity can be increased by either increasing the impulse or by decreasing its mass. In addition to magnetic loading, material ablation on the current- carrying surface of the flyer also provides an impulse upon release of the magnetic pressure. Specifically, as the magnetic pressure decreases to near-zero after peak load, the plasma formed by current diffusion can expand, causing an additional increase in flyer velocity.

Simulations were performed with the MHD code, ALEGRA, to design flyer plates for a given current profile [15]. These calculations resulted in optimal designs for flyer plates of aluminum, copper and titanium that were evaluated experimentally. A major requirement was that the flyer be shocklessly launched to prevent shock heating and thick enough to prevent current diffusion during launch. The 10$\mathrm{mm}$ diameter counter bores in the aluminum anode plates used to carry current, as illustrated in Figure 10. The resulting anode thicknesses ranged from 400 to $725 \mu \mathrm{m}$, where the thinner thicknesses were employed for copper and titanium flyers and the thicker for the aluminum flyers. The copper and titanium flyer plate thicknesses were about $200 \mu \mathrm{m}$. In these experiments, time-resolved velocity 


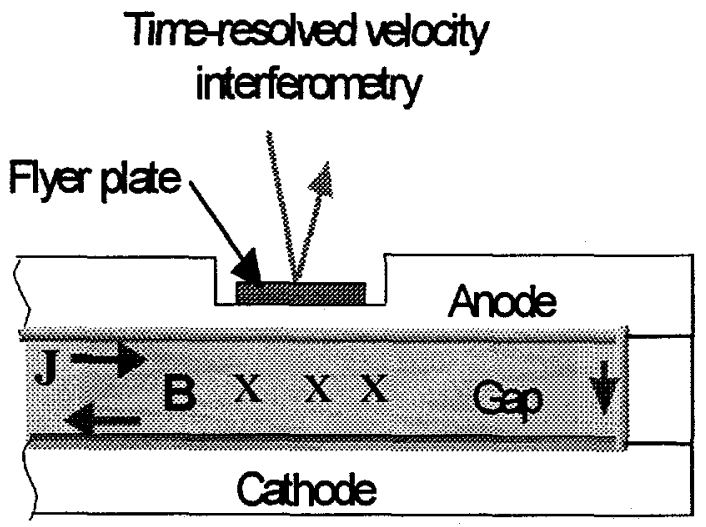

Figure 10. Magnetic loading technique for producing ultra-high velocity flyer plates.

interferometry was used to measure the velocity during the complete launch history.

The results of three experiments on aluminum, titanium and copper are shown in Figure 11. As illustrated, shockless loading of the flyer plates is achieved to final velocities ranging from about 10 to $13 \mathrm{~km} / \mathrm{s}$ in the different materials. This ensures that the flyer plate will not be shock heated during launch and thus retain its initial density and temperature upon impact. Further studies are in progress to determine if other sources of heating, such as joule heating by the current, can produce a change in temperature for the effective thickness of the plate. Initial indications are that the flyer plates are at near-ambient conditions upon impact.

In each experiment illustrated, the flyer thickness was chosen to allow a constant pressure loading history upon impact with a target of about $30 \mathrm{~ns}$ [15]. This time duration is necessary to ensure that a constant pressure is produced on the sample for sufficiently long times to prevent shock alteration. The long pressure durations also result in relatively long transit times in samples and associated EOS accuracy. This technique is being applied to study the high-pressure EOS of several materials, including liquid deuterium [16].

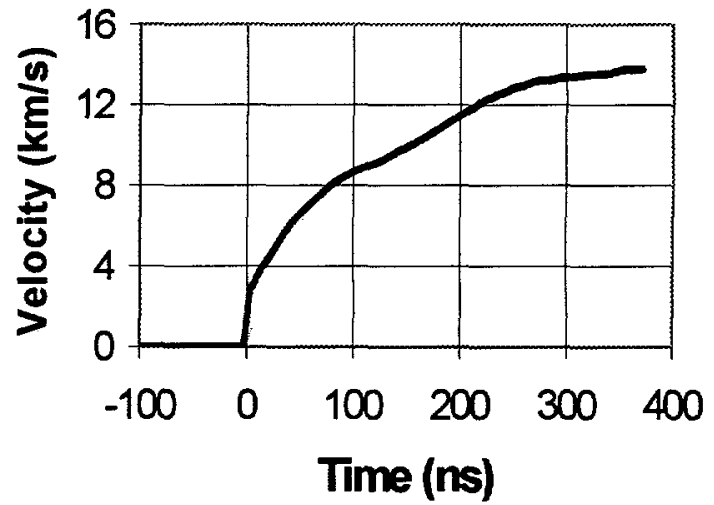

(a)

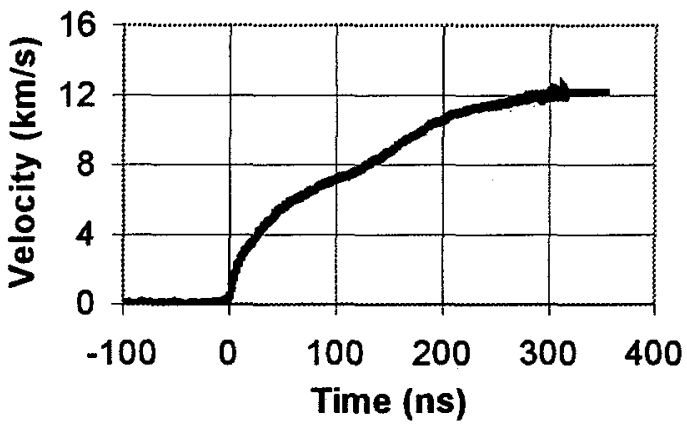

(b)

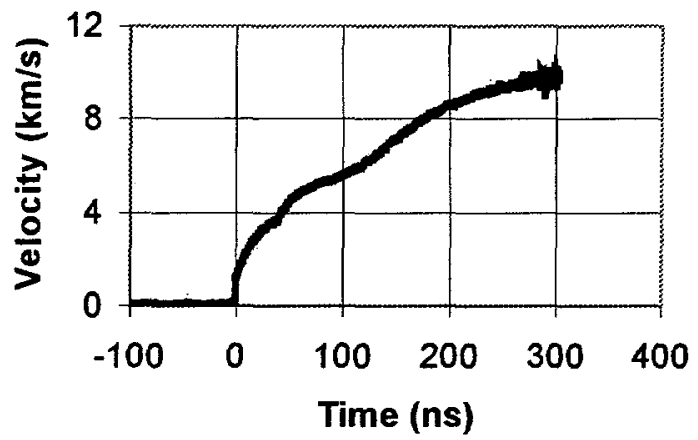

(c)

Figure 11. Magnetically-driven flyer plate velocities. (a) aluminum, (b) titanium, (c) copper. 


\section{Conclusions}

This paper provides a brief review of new experimental developments for producing dynamic isentropic compression of samples to Mbar pressures. Traditional gun launch techniques for producing isentropic compression include use of buffer plates, such as fused silica, to induce ramp waves into specimens mounted on the rear surface of the buffer, or graded density impactors that produce a small amplitude shock followed by continuous compression in a specimen. Both of these approaches have practical limitations. A recent advance in fast pulsed power allows use of magnetic compression to continuously load planar specimens to pressures exceeding $1 \mathrm{Mbar}$ over time scales of approximately $200 \mathrm{~ns}$. Recent experiments using the $\mathrm{Z}$ Accelerator at Sandia indicate that it is possible to isentropically load samples to $400 \mathrm{kbar}$ with accuracy in the pressure-volume-loading curve of about $2 \%$. Experiments on copper and beryllium illustrate the accuracy of the technique.

In addition, the ICE technique is useful for studying dynamic phase transitions, because the ramp waves used to evaluate transition effects do not over-drive phase transitions, as is common in shock wave experiments. Results are presented on iron to illustrate use of the technique. These experiments illustrate that it is possible to determine both the transition stress and the kinetics of the alphaepsilon phase transition in iron in a single experiment.

Finally, ICE wave techniques can be used to launch relatively thick flyer plates to velocities exceeding conventional gas gun methods. Results are presented for aluminum, titanium and copper flyers launched magnetically to velocities of 13,12 and 10 $\mathrm{km} / \mathrm{s}$, respectively. The novel aspect of this approach is that shock waves are not produced in the flyer during launch so that the flyer remains at essentially room temperature. With improvements in progress, this should allow symmetric impact experiments for absolute Hugoniot measurements at pressures of $10 \mathrm{Mbar}$ or more in the laboratory.

\section{Acknowledgements}

We are indebted to several people who have contributed to the development of isentropic loading techniques and the spin-off applications, including Lynn Barker, Lalit Chhabildas, Mike Bernard, Rick Spielman, Bill Stygar, Tim Pointon, Ray Lemke, Brian Oliver, Wayne Trott, Steve Rosenthal, Dennis Hayes, Bob Cauble, Art Toor, and David Reisman.

\section{References}

1. J.R. Asay and G.I. Kerley, Int'l J. Impact Eng'g, 5, 69-99 (1987).

2. G.E. Duvall unpublished, 1968.

3. L.M. Barker and R.E. Hollenbach, J. Appl. Phys. 41, 4208-4226 (1970).

4. L.C. Chhabildas and L.M. Barker, SHOCK WAVES IN CONDENSED MATTER 1987, S.C. Schmidt, N.C. Holmes, editors, Elsevier Sci. Publ, B.V. 111-114 (1988).

5. J.R. Asay and L.C. Chhabildas, HIGH PRESSURE SCIENCE \& TECHNOLOGY, ed. by $\mathrm{B}$. Vodar and $\mathrm{Ph}$. Marteau, Pergamon Press, 2, 958-965 (1980).

6. L.M. Barker, SHOCK WAVES IN CONDENSED MATTER - 1983, J.R. Asay, R.A. Graham, G.K. Straub, editors, Elsevier Sci. Publ, B.V., 217-224 (1984).

7. J.B. Aidun and Y.M. Gupta, J. Appl. Phys. 69, 6998-7014 (1991).

8. M.K. Matzen, Phys. Plasmas. 4 (5), 15191527 (1996).

9. J.R. Asay, in SHOCK COMPRESSION OF CONDENSED MATTER - 1999, ed. by M.D Furnish, L.C. Chhabildas, R.S. Hixson, 1, 261266 (1999).

10. C.A. Hall, Phys. Plasmas 7 (5), 2069-2075 (2000).

11. C.A. Hall, J.R. Asay, W.A. Stygar, R.B. Spielman, S.E. Rosenthal, M.D. Knudson, D.B. Reisman, A. Toor, and R.C. Cauble, submitted to Rev. Scientific Instruments (2000).

12. D.B. Reisman, A. Toor, R.C. Cauble, C.A. Hall, J.R. Asay, M.D. Knudson and M.D. Furnish, submitted to J. Appl. Phys. (2000).

13. L.M. Barker and R.E. Hollenbach, J. Appl. Phys. 45, 4872-4887 (1974). 
14. J.R. Asay, C.A. Hall, K.G. Holland, M.A. Bernard, W.A. Stygar, R.B. Spielman, S.E. Rosenthal, D.H. McDaniel, D.B. Hayes, in SHOCK COMPRESSION OF CONDENSED MATTER - 1999, ed. by M.D Furnish, L.C. Chhabildas, R.S. Hixson, 1, 1151-1154 (1999).

15. C.A. Hall, M.D. Knudson, J.R Asay, R. Lemke, B. Oliver, submitted to J. Impact Engng (2000).

16. M.D. Knudson, private communication (2000). 